\title{
Probing Interactions between Ultracold Fermions
}

\author{
G. K. Campbell ${ }^{1}$, M. M. Boyd ${ }^{1}$, J. W. Thomsen ${ }^{1}$, M. J. Martin ${ }^{1}$, \\ S. Blatt ${ }^{1}$, M. D. Swallows ${ }^{1}$, T. L. Nicholson ${ }^{1}$, T. Fortier ${ }^{2}$, \\ C. W. Oates ${ }^{2}$, S. A. Diddams ${ }^{2}$, N. D. Lemke ${ }^{2}$, P. Naidon ${ }^{3}$, \\ P. Julienne ${ }^{3}$, Jun $\mathrm{Ye}^{1 *}$ \& A. D. Ludlow ${ }^{1}$ \\ ${ }^{1}$ JILA, National Institute of Standards and Technology and University of Colorado \\ Department of Physics, University of Colorado, Boulder, Colorado 80309-0440, USA \\ ${ }^{2}$ Time and Frequency Division, National Institute of Standards and Technology, \\ Boulder, CO 80302, USA \\ ${ }^{3}$ Atomic Physics Division and Joint Quantum Institute, \\ National Institute of Standards and Technology, \\ 100 Bureau Drive Stop 8423, Gaithersburg, Maryland 20899-8423 \\ *To whom correspondence should be addressed; E-mail: junye@jilau1.colorado.edu.
}

\begin{abstract}
At ultracold temperatures, the Pauli exclusion principle suppresses collisions between identical fermions. This has motivated the development of atomic clocks using fermionic isotopes. However, by probing an optical clock transition with thousands of lattice-confined, ultracold fermionic $\mathrm{Sr}$ atoms, we have observed density-dependent collisional frequency shifts. These collision effects have been measured systematically and are supported by a theoretical description attributing them to inhomogeneities in the probe excitation process that render the atoms distinguishable. This work has also yielded insights for zeroing the clock density shift.
\end{abstract}

Quantum statistics play a critical role in shaping interactions between matter. This is apparent in the markedly different behavior of Bose-Einstein condensates $(1,2)$ and degenerate 
Fermi gases of ultracold atoms (3). The quantum statistics of atoms can thus be a key factor in the choice of an atomic system for a given experiment. Such is the case for atoms at the heart of an atomic clock. Simultaneous interrogation of many atoms is favorable for achieving high measurement precision. However, when atoms interact with each other, their internal energy states can be perturbed, leading to frequency shifts of the clock transition $(4,5)$. The use of identical fermions was prescribed to allow many atoms to strengthen the signal without such density-dependent collision shifts (6). Previous experiments seemed to confirm this fact for both single-component (7) and two-component fermion mixtures (8).

However, by probing an optical clock transition with thousands of fermionic Sr atoms confined in a one-dimensional optical lattice, we clearly observe density-related frequency shifts at a fractional precision of $1 \times 10^{-16}$. When the light-atom interaction introduces a small degree of inhomogeneous excitation, previously indistinguishable fermions become slightly distinguishable. This effect causes a time-dependent variation of the two-particle correlation function, giving rise to an apparent mean-field energy. The resulting collision effects have been measured systematically as a function of temperature, excitation probability, and interaction inhomogeneity. These observations are supported by a theoretical description of fermionic interactions including the effect of the measurement process.

The latest generation of optical atomic clocks such as those based on the ${ }^{1} \mathrm{~S}_{0}-{ }^{3} \mathrm{P}_{0}$ transition in fermionic ${ }^{87} \mathrm{Sr}$ currently offers the highest measurement precision, useful for measuring possible atomic interactions $(9,10)$. In an ultracold dilute gas with a mean field energy, a narrow clock transition will experience a density-dependent frequency shift $(11,12)$ given by $h \Delta \nu=\left(4 \pi \hbar^{2} G^{(2)} \rho a\right) / m$. Here $m$ is the atomic mass, $\rho$ is the density of the atomic sample, $a$ is the $s$-wave scattering length characterizing the atomic interaction, and $h=2 \pi \hbar$ is Planck's constant. $G^{(2)}$ is the two-atom correlation function at zero distance, which summarizes the quantum statistics of colliding bodies. For example, $G^{(2)}=0$ for identical fermions 
and $G^{(2)}=2$ for identical bosons in a thermal gas. The Fermi suppression arises from the Pauli exclusion principle, which prohibits even-partial-wave collisions between indistinguishable fermions. At ultracold temperatures partial waves higher than $s$-wave are frozen out (13). For atoms excited in our two-level clock system, three possible $s$-wave interactions exist: those between two ${ }^{1} \mathrm{~S}_{0}$ ground-state $(|g\rangle)$ atoms, those between two ${ }^{3} \mathrm{P}_{0}$ excited-state $(|e\rangle)$ atoms, and those between a $|g\rangle$ atom and a $|e\rangle$ atom. Including all possible interactions, the collisional frequency shift at ultracold temperatures is given by Eq. $1(8,12,11)$ :

$$
\Delta \nu_{g e}=\frac{2 \hbar}{m}\left(G_{g e}^{(2)} a_{g e}\left(\rho_{g}-\rho_{e}\right)+G_{e e}^{(2)} a_{e e} \rho_{e}-G_{g g}^{(2)} a_{g g} \rho_{g}\right),
$$

where $a_{i j}$ is the $s$-wave scattering length for collisions between atoms in state $i$ and $j$, and $\rho_{i}$ is the density of atoms in state $i$. Since indistinguishable fermions do not collide, $G_{g g}^{(2)}=G_{e e}^{(2)}$ $=0$. Fermions in different internal states are distinguishable, and for a completely incoherent mixture of the two states, $G_{g e}^{(2)}=1$. However, if the two-state mixture is prepared by a uniform, coherent excitation of ground-state atoms, then the fermions evolve indistinguishably and $G_{g e}^{(2)}$ $=0(8)$. In this case, $\Delta \nu_{g e}=0$.

Two possibilities exist for $\Delta \nu_{g e}$ to deviate from zero. First, the $p$-wave contribution may not be negligible. However, for ultracold atoms confined in a well-characterized optical trap, we show experimental evidences and theoretical calculations that conclude that $p$-wave collisions make no noticeable contribution to the observed clock frequency shift. Second, it is imperative to consider the entire interaction, including the measurement process, when exploring the question of whether fermions collide. In fact, the measurement process, such as probing a clock transition, may strongly influence the time-dependent variations of $G^{(2)}$. We show here that an inhomogeneous interaction between light and atoms leads to the loss of indistinguishability of the fermions, thus making $0<G^{(2)}<1$.

Although a uniform, coherent excitation of identical fermions maintains $G^{(2)}=0$, and no 
$s$-wave collisions occur, if a small non uniformity in the excitation process arises, the atoms are no longer completely identical, and $G^{(2)}>0$. The value of $G^{(2)}$ will depend on the degree of excitation inhomogeneity. This measurement-induced dynamic variation of quantum statistics leads directly to a change of the mean-field energy within the ultracold gas, resulting in a nonzero $\Delta \nu_{g e}$. It is interesting to contrast the present work with previous results observed using an ultracold gas of fermionic ${ }^{6} \mathrm{Li}$, where the insensitivity of a radio-frequency transition to collisional shifts was demonstrated $(7,8)$. It was shown that the fermionic insensitivity to collisional shifts was maintained even when a pure superposition state of the two level system had decohered. This decoherence would have allowed interactions, but when a uniform rf probing field reintroduced coherence to the atoms in a homogeneous manner, $G^{(2)}$ again became zero, giving no collisional shifts within the measurement precision (14). ¿From the current experiment it is clear that any non-identical evolutions during the interrogation process lead to the breakdown of Fermi suppression; this experiment is sensitive to very small inhomogeneities because of the high measurement precision.

An intuitive understanding emerges from considering two sample atoms in a pseudo spin$1 / 2$ system with ground $|g\rangle$ and excited $|e\rangle$ states. Before applying the spectroscopy pulse, the atomic system is in a pure, polarized spin state with $\left|\psi_{1}\right\rangle=\left|\psi_{2}\right\rangle=|g\rangle$. The effect of the pulse is to perform a rotation on the Bloch sphere, as shown in the inset of Fig. 1B. For a coherent, homogeneous excitation, the wavefunction of the system becomes a coherent superposition $\left|\psi_{1}\right\rangle=\left|\psi_{2}\right\rangle=\alpha|g\rangle+\beta|e\rangle$. The wavefunctions of both atoms are identical, $G_{12}^{(2)}=0$, and collisions cannot occur. An inhomogenous spectroscopic excitation, such as that caused by varying Rabi frequencies for different atoms, results in slightly different rotations on the Bloch sphere for the two atoms (Fig. 1B inset). Hence we have $\left|\psi_{1}\right\rangle=\alpha|g\rangle+\beta|e\rangle$ and $\left|\psi_{2}\right\rangle=$ $\gamma|g\rangle+\delta|e\rangle$. The fermions are distinguishable and $0<G_{12}^{(2)}<1$. The value of $G_{12}^{(2)}$ depends on the amount of inhomogeneity, and its time variation can be explicitly calculated from the 
anti-symmetrized overlap of the two wavefunctions (details in supporting text (15)):

$$
G_{12}^{(2)}(\alpha(t), \beta(t), \gamma(t), \delta(t))=1-\left|\alpha(t) \gamma^{*}(t)+\beta(t) \delta^{*}(t)\right|^{2}
$$

The resulting collision shift from Eq. 1 is then

$$
\Delta \nu(t)=\frac{2 \hbar a_{g e}}{m} G_{12}^{(2)}(\alpha, \beta, \gamma, \delta)\left(\rho_{g}-\rho_{e}\right)
$$

Before proceeding with experimental results, we first summarize the system under study (15). In the ${ }^{87} \mathrm{Sr}$ optical clock, atoms are trapped in a one-dimensional optical standing-wave potential (1D optical lattice). Longitudinally the atoms are confined tightly, with an oscillation frequency $\nu_{z} \sim 80 \mathrm{kHz}$. At temperature $T=1 \mu \mathrm{K}, \sim 98 \%$ of the atoms occupy the groundstate of the trap $\left(\bar{n}_{z}=0.02\right)$. The laser probing the clock transition propagates along the lattice axis, and spectroscopy is performed in the Lamb-Dicke regime. In the transverse plane the confinement is much weaker, with an oscillation frequency $\nu_{x}=\nu_{y} \sim 450 \mathrm{~Hz}$, and atoms occupy a large number of motional states $\left(\bar{n}_{x}=\bar{n}_{y}=46\right)$. Typically, $\sim 2 \times 10^{3}$ atoms are trapped in the optical lattice, resulting in 30 atoms per lattice site with a density of $2 \times 10^{11} \mathrm{~cm}^{-3}$ (15). The optical lattice is nearly vertically oriented and is operated at the so called "magic wavelength" of $\lambda_{L} \sim 813.429 \mathrm{~nm}(16)$, where the ac Stark shifts of the ${ }^{1} \mathrm{~S}_{0}$ and ${ }^{3} \mathrm{P}_{0}$ states are identical.

With a perfect alignment of the probe laser along the strong confinement axis, assuming cylindrical symmetry, a residual angular spread between the probe and lattice $\vec{k}$ remains due to the finite size of the lattice beam (17). However, an even larger effect occurs if the symmetry is broken due to either aberrations in the beam profile or angular misalignment $(\Delta \theta)$ between the lattice and the probe beam. For our trap parameters, we estimate an effective $\Delta \theta \approx 10 \mathrm{mrad}$ (Fig.1A inset). The residual wave-vector projected on the transverse plane leads to slightly different excitation Rabi frequencies $\Omega_{\vec{n}}$ for atoms in different $\left(n_{x}, n_{y}\right)$ states $(15,18,19)$. For a given $T$, the occupation of a transverse motional state $n_{x, y}$ is given by the normalized Maxwell- 
Boltzmann distribution. The inhomogeneity in the Rabi frequencies is thus affected by both $T$ and $\Delta \theta$.

To calculate the density shift, we return to our two-atom model. Each atom has a slightly different $\Omega_{\vec{n}}$. For the entire atomic ensemble, we can define an average Rabi frequency $\bar{\Omega}$ and its RMS spread $\Delta \Omega$. To approximate the average density shift, we set $\Omega_{1}=\bar{\Omega}+\Delta \Omega$ and $\Omega_{2}=\bar{\Omega}-\Delta \Omega$ for our two-atom model. Thus the time-dependent quantities, $\alpha, \beta, \gamma, \delta$ as defined in Eq. 2 are parameterized by $\bar{\Omega}$ and $\Delta \Omega$ (15). At a time $t$ during the spectroscopy pulse, the atoms experience an ensemble-averaged shift:

$$
\Delta \nu(t)=\frac{2 \hbar a_{g e}}{m} G_{12}^{(2)}(\bar{\Omega}+\Delta \Omega, \bar{\Omega}-\Delta \Omega)\left(\rho_{g}-\rho_{e}\right) .
$$

This shift evolves during the spectroscopy pulse, and for the final density shift we time average $\Delta \nu(t)$ over the total pulse length $t_{F}$. This approximation is valid in the limit that the change in $\Omega$ due to atomic interactions is much less than $\Delta \Omega$. A more rigorous calculation using the optical Bloch equations including atomic interactions has also been made. Using our typical trap parameters we find the two-atom approximation is valid to within $5 \%$. The time-dependent Rabi-oscillation is only slightly affected by atomic interactions, however the effect on the final clock shift is obvious.

For inhomogeneity-induced collision shifts, $t_{F}$ is important. Atoms in close proximity to each other tend to have similar Rabi frequencies, whereas atoms located far apart are more likely to experience different excitations (and hence be distinguishable). If $t_{F} \nu_{x, y} \ll 1$, the atoms are effectively frozen in place, and will experience no density shift. However, if $t_{F} \nu_{x, y}>1$, atoms initially located far apart have time to interact. For the clock experiment requiring high spectral resolution, we have $t_{F}=80 \mathrm{~ms}$ and $1 / \nu_{x, y}=2.2 \mathrm{~ms}$, so collisions will occur.

To systematically study these effects, we implemented controlled variations of both $T$ and $\Delta \theta$. To vary $T$, we perform cooling (heating) of the lattice-confined atoms in three dimensions: 
Doppler cooling (heating) along the transverse direction and sideband cooling (heating) along the longitudinal axis. Simultaneous with the sideband cooling (heating), the atoms are spinpolarized by optical pumping in a weak magnetic $(B)$ bias field. Atoms are polarized into either the $m_{F}=+9 / 2$ or $m_{F}=-9 / 2$ Zeeman states. The ${ }^{1} \mathrm{~S}_{0}-{ }^{3} \mathrm{P}_{0}$ clock transition, which is predicted to have a natural linewidth of $\sim 1 \mathrm{mHz}(20,21,22)$, is interrogated using a cavity-stabilized diode laser at $698 \mathrm{~nm}$ with a linewidth below $1 \mathrm{~Hz}$ (23). Spectroscopy is performed in the LambDicke regime and in the resolved sideband limit (24). To ensure that the polarized spin state is well resolved from other $m_{F}$ levels, spectroscopy is performed under $B \sim 250 \mathrm{mG}$, leading to a separation of $250 \mathrm{~Hz}$ between the $m_{F}= \pm 9 / 2$ states. A spectroscopy pulse length of $t_{F}=80$ ms results in a Fourier-limited linewidth of $\sim 10 \mathrm{~Hz}$.

After the spectroscopy pulse is applied, atoms remaining in $|g\rangle$ are counted by measuring fluorescence on the strong ${ }^{1} \mathrm{~S}_{0}-{ }^{1} \mathrm{P}_{1}$ transition. Atoms transferred to $|e\rangle$ are then pumped back to $|g\rangle$ via the intermediate $(5 \mathrm{~s} 6 \mathrm{~s})^{3} \mathrm{~S}_{1}$ states and are also counted. Combining these two measurements gives us a normalized excitation fraction $\rho_{e} /\left(\rho_{e}+\rho_{g}\right)$. The atomic temperature is determined using both sideband spectroscopy $(25,15)$ and time-of-flight analysis. In Fig. 1A, sample spectra are shown for two different values of $T$. Once $T$ is measured, the degree of inhomogeneity is determined by fitting the decaying Rabi oscillations for the ensemble. In Fig. 1B, the Rabi oscillation at $T=3 \mu \mathrm{K}$ (squares) clearly shows faster dephasing than that of $T=1$ $\mu \mathrm{K}$ (circles), indicating a larger degree of inhomogeneity.

Density-dependent frequency shifts of the ${ }^{87} \mathrm{Sr}$ clock transition are measured using a remotely located calcium optical standard at NIST (9) as a stable frequency reference, which is linked to JILA via a phase-coherent fiber network (26). This direct optical frequency measurement between two optical standards allows fractional measurement precision of a few times $10^{-16}$ after hundreds of seconds of averaging. To measure the clock center frequency, the spectroscopy pulse is first applied to atoms optically pumped to the $m_{F}=+9 / 2$ state. In the next 
cycle, atoms polarized to the $m_{F}=-9 / 2$ state are used. The center frequency is then determined by the average of both resonances. The density-dependent frequency shift is determined using an interleaved scheme, where the density of the atomic ensemble is varied every $100 \mathrm{~s}$. The density is varied by a factor of two. Pairs of such data are then used to measure a frequency shift, and many pairs are averaged to decrease the statistical uncertainty. Typically, we lock the clock laser near the full-width at half-maximum of each resonance, however the location of the lock points is varied to select the desired excitation fraction.

Spectroscopy is performed using two different experimental procedures. In the first, we probe the clock transition from $|g\rangle$ to $|e\rangle$ (Fig. 2 inset). The intensity of the probe is set to produce a $\pi$-pulse on resonance. This direct scheme could suffer from imperfect polarization of the atomic sample, and spectator atoms could be left in other $m_{F}$ levels. This scenario could potentially lead to density-dependent shifts due to collisions between different $m_{F}$ states that are not suppressed by the Fermi statistics. The second scheme minimizes this effect by probing $|e\rangle$ to $|g\rangle$ (Fig. 2). Here we apply a strong pulse to first transfer the population from $|g\rangle$ to $|e\rangle$. The pulse power broadens the transition in order to decrease the sensitivity of population transfer to probe laser frequency, and transfers $\sim 50 \%$ of the population to $|e\rangle$. This first pulse is resonant with atoms in one of the $m_{F}= \pm 9 / 2$ states, hence atoms left in other $m_{F}$ states due to imperfect polarization are not transferred. Subsequently, all atoms remaining in $|g\rangle$ are removed from the lattice with a pulse of light resonant with the strong ${ }^{1} \mathrm{~S}_{0}-{ }^{1} \mathrm{P}_{1}$ transition, without affecting the temperature of the atoms in $|e\rangle$. This is confirmed with sideband spectroscopy (15). Finally, the clock transition of $|e\rangle$ to $|g\rangle$ is probed with the usual $80 \mathrm{~ms} \pi$-pulse. In both experimental procedures, we measure populations in $|e\rangle$ and $|g\rangle$ to determine the normalized excitation fraction.

Figure 2 summarizes the measured density-dependent frequency shift as a function of the normalized ground-state fraction for two different values of $T, 1 \mu \mathrm{K}$ (squares) and $3 \mu \mathrm{K}$ (cir- 
cles). The data indicate a clear trend that the density shift decreases under a more homogeneous excitation. The solid lines are the expected shifts calculated from the two-atom model. For clock operation, it is important to note that near $50 \%$ excitation fraction, for both $T$, the shift goes through zero.

Of course, as we change $T$, we vary both the excitation inhomogeneity and the $p$-wave contribution. To estimate the magnitude of $p$-wave collisions, we note that the van der Waals potential for all three interaction types ( $g g, e e$, or $e g$ ) has been theoretically calculated (27, 21,28 ), and the $p$-wave centrifugal barrier is expected to be greater than $25 \mu \mathrm{K}$. At $T \sim 1 \mu \mathrm{K}$, $k a \ll 1$, where $k=2 \pi / \lambda_{T} \cdot \lambda_{T}=h / \sqrt{2 \pi m k_{B} T}$ is the thermal deBroglie wavelength, and $k_{B}$ is the Boltzmann constant. Under these conditions, the ratio of $p$-wave to $s$-wave phase shift is $(b k)^{2} b / a$, where $b$ is the $p$-wave scattering length. For $g g$ interactions, the $s$-wave scattering length has been measured (29) for ${ }^{88} \mathrm{Sr}$, and mass scaling gives $a_{g g}=96.2(1) a_{0}$ for ${ }^{87} \mathrm{Sr}$, where $a_{0}$ is the Bohr radius. Combined with the van der Waals potential, the $p$-wave phase shift can be determined from the Schrödinger equation. For ${ }^{1} \mathrm{~S}_{0}, b_{g g}=-76 a_{0}$, and for $T=1 \mu \mathrm{K}$, $\left|\left(b_{g g} k\right)^{2} b_{g g} / a_{g g}\right| \approx 0.01$. Thus, $p$-wave collisions for $g g$ are suppressed by over two orders of magnitude and are negligibly small. Although the $s$-wave scattering lengths $a_{e e}$ and $a_{g e}$ have not yet been measured and thus cannot directly constrain the values of $b_{e e}$ and $b_{e g}$, calculations based on a theoretical potential predict that these $p$-wave collisions are similarly suppressed relative to $s$-wave collisions. An exception would be a $p$-wave shape resonance (13); however, this would occur only for a very small range of possible $a_{e e}$ and $a_{g e}$, and the effect would be reduced by thermal averaging. We also note that in a trapping potential, $k$ is modified due to the zero-point energy of the trap $\left(k_{Z P}\right)$ and the effective thermal wavevector for collisions is given by $k_{T}=\sqrt{\left(k^{2}+k_{Z P}^{2}\right) / 2}$. For our trap, $k_{Z P} \sim 3.5 \mu \mathrm{K}$, and $p$-wave collisions are still suppressed. The observed density shift scales as $G_{12}^{(2)} a_{g e}$, and for our typical temperatures we find values of $G_{12}^{(2)}$ between 0.03 and 0.15 , whereas the $p$-wave scattering length is expected to 
be $\sim 1 \%$ of $a_{g e}$. Hence, inhomogeneity-induced $s$-wave collisions dominate. In the the unitarity limit where $k_{T}\left|a_{g e}\right|>1$ ( $a_{g e}$ is the zero-temperature scattering length), the effective scattering length is $1 / k_{T}$. For our lattice trap parameters and temperature range of $1-3 \mu \mathrm{K}$, this length is on the order of $-300 a_{0}$, which is consistent in sign and magnitude with our observed frequency shifts, along with the values and uncertainties of $G_{12}^{(2)}$ and $\rho$.

To provide further evidence to exclude $p$-wave contributions, we vary the inhomogeneity by misalignment of the spectroscopy probe beam under a fixed $T$. This also helps rule out $\bar{n}_{x, y, z}$ - dependent residual ac Stark shift of the trap. Typically the probe beam is coaligned with the lattice to minimize motional effects. However, by increasing the misalignment $(\Delta \theta)$, we can also increase $\Delta \Omega$. Fig. 3A and B show Rabi oscillations for two different probe beam misalignments at $T=1 \mu \mathrm{K}$ (triangles and open squares) and $3 \mu \mathrm{K}$ (circles and open diamonds), respectively. Fig. 3C displays the measured density shift as a function of $(\Delta \Omega / \bar{\Omega})$ due to probe misalignment. For $T=1 \mu \mathrm{K}$, the shift becomes larger with increased $\Delta \Omega / \bar{\Omega}$. When $\Delta \Omega / \bar{\Omega}$ increases further, the $3 \mu \mathrm{K}$ data indicate that the density shift becomes smaller. This behavior is reproduced by the theoretical curves shown in Fig. 3C, and is illustrated in Fig. 3D. Consider two different $\Delta \Omega / \bar{\Omega}$, both with an average excitation fraction of 0.3 . In the first case, for small misalignment, we find a spread in the excitation fraction of \pm 0.2 ; there is an inhomogeneity allowing collisions to occur and we measure a small density shift. In the second case, with further misalignment the spread in the excitation fraction increases to \pm 0.4 ; there is now a larger spread in the Rabi frequencies, and collisions still occur. However, we now have atoms with an excitation fraction both above and below $50 \%$ where the shift crosses zero. Hence, the collisions of atoms with excitations between $0.3-0.7$ will average to zero (this is consistent with the density shift going to zero at $50 \%$ excitation, regardless of the inhomogeneity), and the final collision shift is due only to atoms with excitation fractions between 0 and 0.3 . The measured shift for the larger misalignment is therefore smaller. 
Combining the measurements shown in Figs. 2 and 3 makes it clear that the observed density-dependent shifts arise from the change of the quantum statistics $G^{(2)}$ caused by the inhomogeneous measurement process. Of course, the inhomogeneous effect can be suppressed by decreasing the sample temperature and increasing the transverse confinement, or going to higher dimension traps. Importantly, for clock operations we have identified that near a $50 \%$ excitation fraction the density shift goes to zero. Using these measurements we can now reduce the uncertainty of the collision shifts for clock operation (9) to $5 \times 10^{-17}$. This time dependent variation in quantum statistics will also apply to boson-based clocks, where the original $G^{(2)}=$ 2 will decrease to a value between 1 and 2 .

\section{References}

1. E. A. Cornell, C. E. Wieman, Rev. Mod. Phys. 74, 875 (2002).

2. W. Ketterle, Rev. Mod. Phys. 74, 1131 (2002).

3. B. DeMarco, D. S. Jin, Science 285, 1703 (1999).

4. K. Gibble, S. Chu, Phys. Rev. Lett. 70, 1771 (1993).

5. Y. Sortais, et al., Phys. Scr. T95, 50 (2001).

6. K. Gibble, B. J. Verhaar, Phys. Rev. A 52, 3370 (1995).

7. S. Gupta, et al., Science 300, 1723 (2003).

8. M. W. Zwierlein, Z. Hadzibabic, S. Gupta, W. Ketterle, Phys. Rev. Lett. 91, 250404 (2003).

9. A. D. Ludlow, et al., Science 319, 1805 (2008).

10. G. K. Campbell, et al., Metrologia 45, 539 (2008). 
11. P. J. Leo, P. S. Julienne, F. H. Mies, C. J. Williams, Phys. Rev. Lett. 86, 3743 (2001).

12. D. M. Harber, H. J. Lewandowski, J. M. McGuirk, E. A. Cornell, Phys. Rev. A 66, 053616 (2002).

13. B. DeMarco, J. L. Bohn, J. P. Burke, M. Holland, D. S. Jin, Phys. Rev. Lett. 82, 4208 (1999).

14. To mitigate the effects of inhomogeneous line broadening due to the density distribution of trapped atoms, the MIT experiment spatially selected only a small central region when measuring the excitation fraction. By spatially selecting only a small fraction of trapped atoms where the trapping potential is more homogenous, the MIT group minimized their sensitivity to the inhomogeneous evolution described in this experiemnt.

15. Materials and methods are detailed in supporting online material available at Science Online.

16. J. Ye, H. J. Kimble, H. Katori, Science 320, 1734 (2008).

17. P. J. Martin, B. G. Oldaker, A. H. Miklich, D. E. Pritchard, Phys. Rev. Lett. 60, 515 (1988).

18. D. J. Wineland, W. M. Itano, Phys. Rev. A 20, 1521 (1979).

19. T. Akatsuka, M. Takamoto, H. Katori, Nature Physics 4, 954 (2008).

20. M. M. Boyd, et al., Phys. Rev. A 76, 022510 (2007).

21. R. Santra, K. V. Christ, C. H. Greene, Phys. Rev. A 69, 042510 (2004).

22. S. G. Porsev, A. Derevianko, Phys. Rev. A 69, 042506 (2004).

23. A. D. Ludlow, et al., Optics Letters 32, 641 (2007).

24. D. Leibfried, R. Blatt, C. Monroe, D. Wineland, Rev. Mod. Phys 75, 281 (2003). 
25. By analyzing the spectral components in sideband spectroscopy, the longitudinal temperature can be accurately determined. Extracting the transverse temperature is more complicated, however we have confirmed using time-of-flight analysis that the transverse and longitudinal temperatures are identical both before and after cooling (heating).

26. S. M. Foreman, et al., Phys. Rev. Lett. 99, 153601 (2007).

27. S. G. Porsev, A. Derevianko, Phys. Rev. A 65, 020701 (2002).

28. We have calculated the phase shifts, and corresponding lengths, using a model S+P potential with variable short-range shapes to change the scattering length over its full range. The short-range shape parameter varies so as to change the threshold phase and scattering length, corresponding approximately to changing the number of bound states in the potential by one. This represents the possible ranges of variation of any $\mathrm{Sr}$ van der Waals potential.

29. Y. N. M. de Escobar, et al., Arxiv preprint arXiv:0808.3434v1 (2008).

30. We greatly appreciate technical contributions of T. Zelevinsky and insightful discussions with K. Gibble. We acknowledge funding support from NIST, NSF, ONR, and DARPA. G. Campbell and A. D. Ludlow are supported by National Research Council postdoctoral fellowships. A.D.L.'s present address is NIST Time and Frequency Division. J. W. Thomsen is a JILA visiting fellow, with a permanent address: The Niels Bohr Institute, Universitetsparken 5, 2100 Copenhagen, Denmark. The current address for P. Naidon is ERATO Macroscopic Quantum Project, JST, Tokyo, 113-0033 Japan. 
A
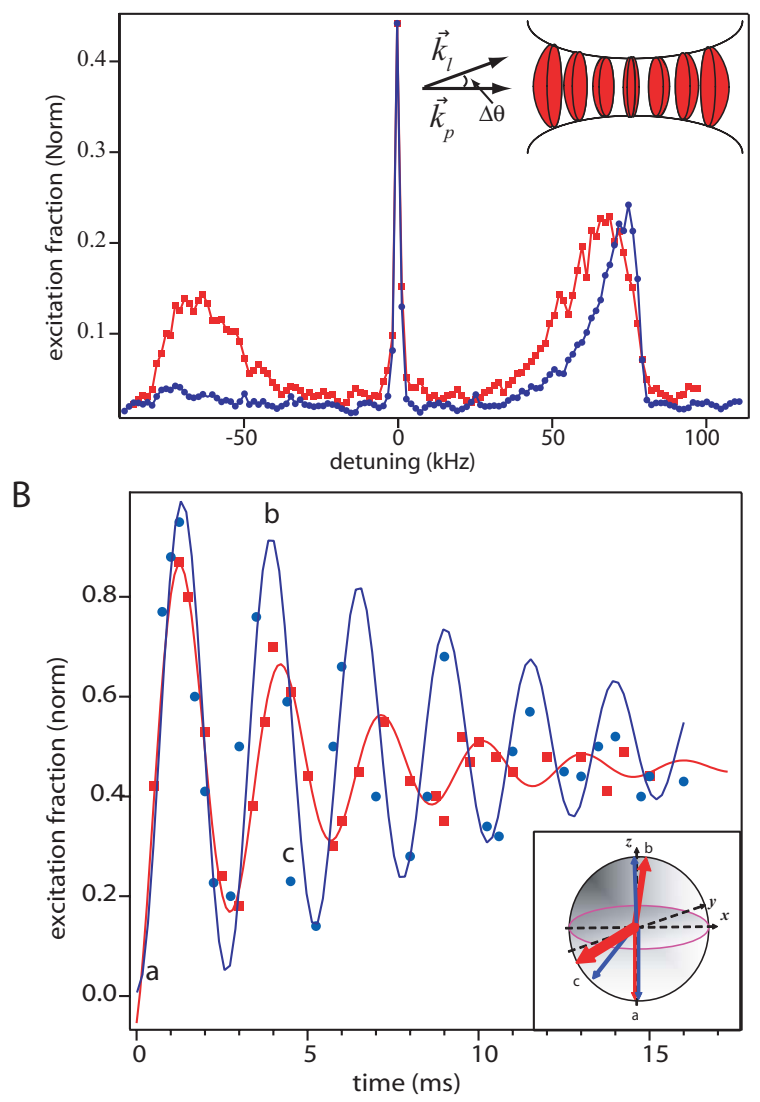

Figure 1: (A) Sideband excitation spectra for $T=1 \mu \mathrm{K}$ (blue circles) and $3 \mu \mathrm{K}$ (red squares). The spectra are obtained in the resolved sideband limit and have three dominant features, the narrow carrier transition and broad red (blue) motional sidebands that are excited when an atom is transferred to a lower (higher) motional state during the transition. As the temperature of the sample is lowered, the atoms primarily occupy the ground-state and the red sideband is suppressed. The temperature of the atomic ensemble can be extracted from a fit of the sidebands (25). The inset shows the lattice geometry and excitation scheme. The probe beam and lattice are coaligned and copolarized, minimizing the relative spread between $\overrightarrow{k_{l}}$ and $\overrightarrow{k_{p}}$. However, even under the best effort, a small angle $\Delta \theta$ between the probe and lattice beams may persist due to aberrations and misalignment. (B) Rabi oscillations for temperatures of $1 \mu \mathrm{K}$ (blue circles) and $3 \mu \mathrm{K}$ (red squares). For higher temperatures, more motional states are occupied. This leads to a larger spread in the Rabi frequencies and faster dephasing of the excitation between atoms. By fitting the decay of Rabi oscillations we can determine the degree of excitation inhomogeneity. The inset illustrates the dephasing process using rotations on the Bloch sphere. At time a, before the excitation, the atoms are in a pure state. At time $b$, the atoms have undergone two oscillations. For the red curve the temperature is hotter and there is a larger spread in Rabi frequencies. This is indicated by the increased width of the Bloch vector and dephasing of the observed oscillations. At time $\mathrm{c}$, the effect is even more pronounced. 


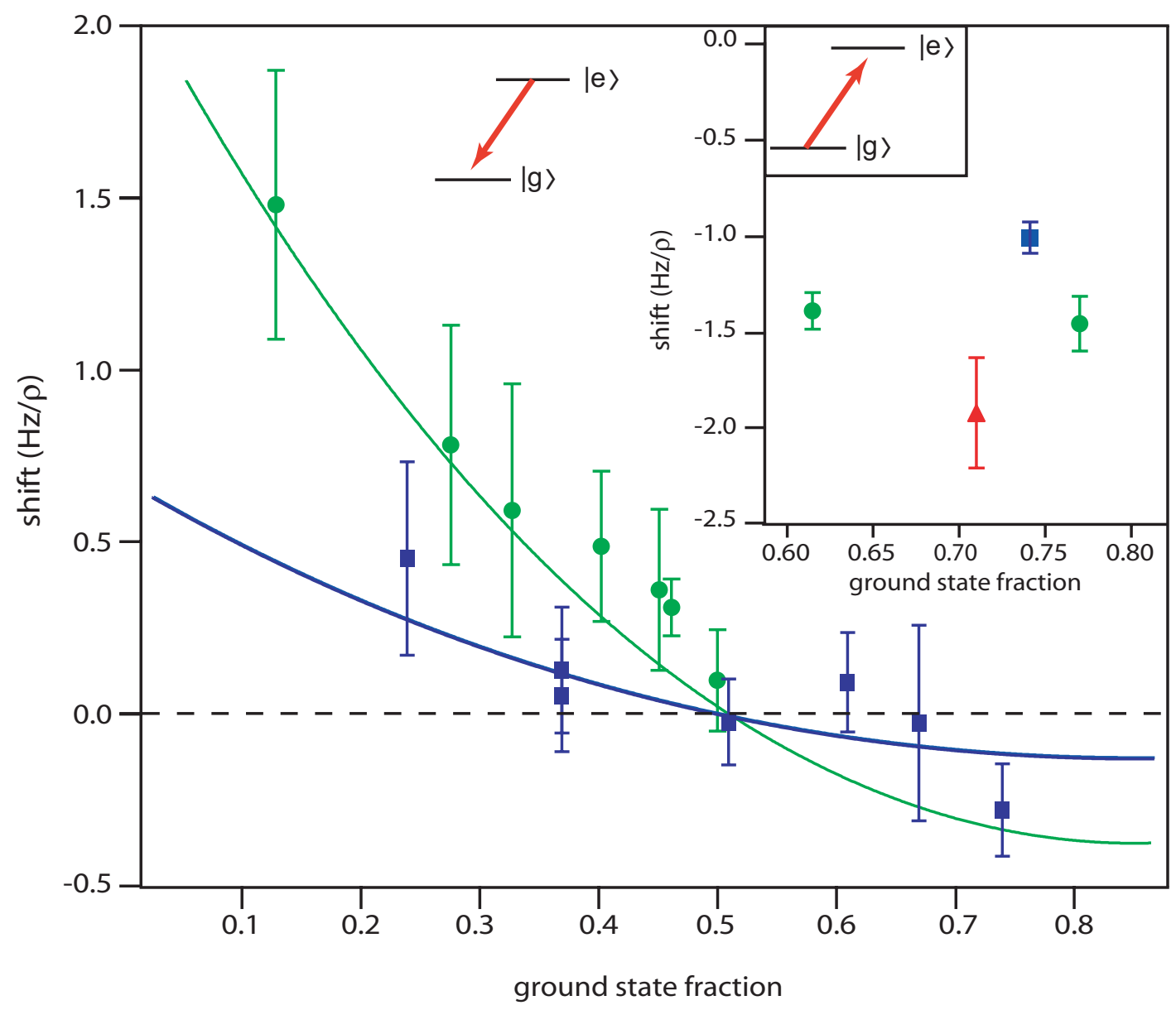

Figure 2: Measured density-dependent frequency shift as a function of the final excitation fraction and temperature. Atoms are initially spin polarized and transferred to ${ }^{3} \mathrm{P}_{0}(|e\rangle)$ before the spectroscopy pulse is applied. The squares (circles) show the measured shift for $T=1$ (3) $\mu \mathrm{K}$. The lines show the calculated shifts using the two-atom model with only a single scaling factor. Near $\sim 50 \%$ the shift goes through zero. In the inset, the measured shift is shown for atoms excited from the ${ }^{1} \mathrm{~S}_{0}(|g\rangle)$ state for $T=1 \mu \mathrm{K}$ (squares), $3 \mu \mathrm{K}$ (circles), and $5 \mu \mathrm{K}$ (triangles). However, the magnitude in this case could be influenced by imperfect spin polarizations. For both plots, as the temperature is decreased, the inhomogeneity also decreases, leading to a smaller collision shift. $\rho$ is the atomic density of $10^{11} / \mathrm{cm}^{3}$. The density dependent shift for each excitation fraction is determined using an interleaved scheme where the density is varied every $100 \mathrm{~s}$. Pairs of such data are then used to determine the frequency shift. Typical data sets include 20 - 30 pairs of density comparison, with the error bars indicating the standard error. 
A

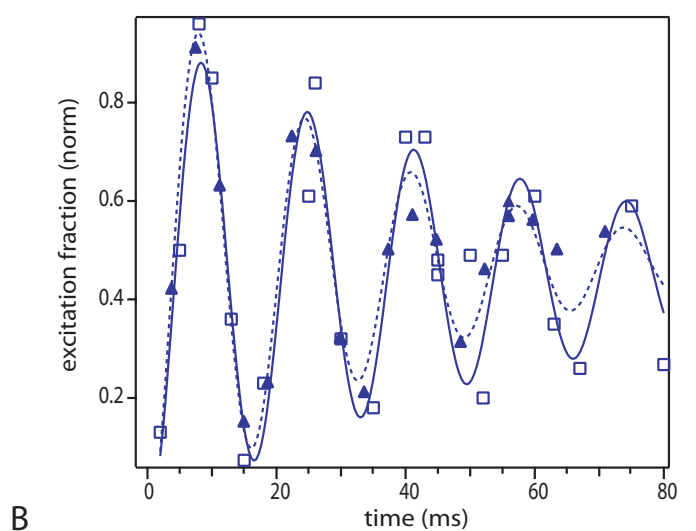

B

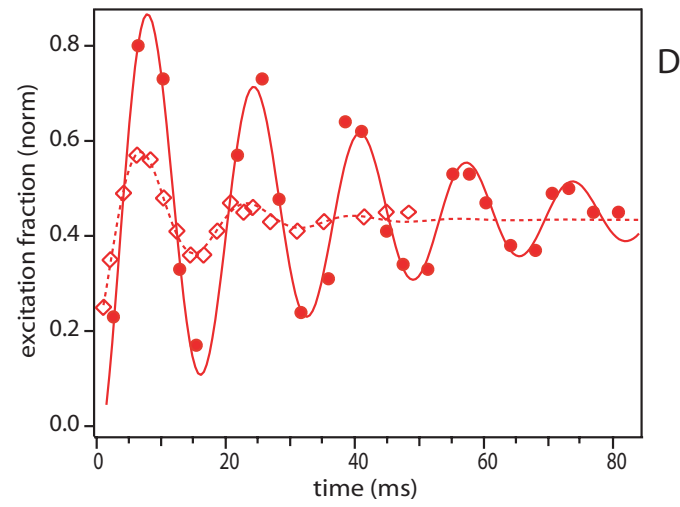

C

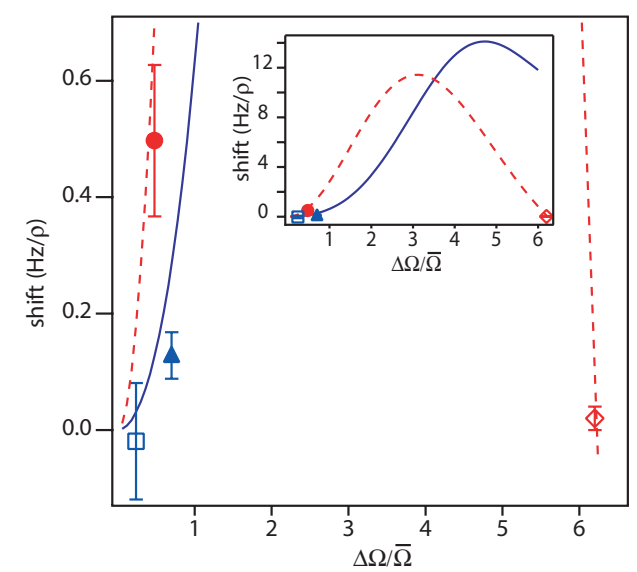

D
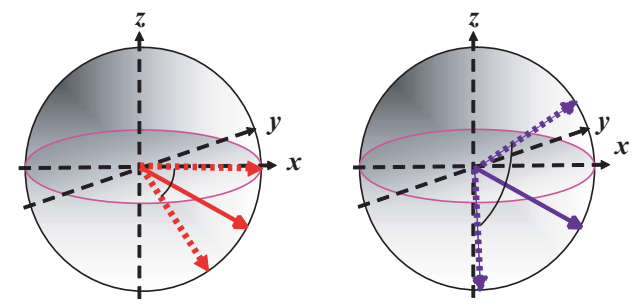

Figure 3: Effect of probe misalignment on the density-dependent shift. (A) Rabi oscillations are shown for two different values of $\Delta \theta$ at $T=1 \mu \mathrm{K}$. The open squares show oscillations when the probe is aligned similar to that of Fig. 1 and 2. The solid triangles show a faster dephasing when the probe beam misalignment is increased further by $5 \mathrm{mrad}$. (B) Rabi oscillations for $T$ $=3 \mu \mathrm{K}$. The circles show oscillations when the probe beam is aligned similar to that of Fig. 1 and 2 , and the diamonds when the misaligment is increased further by $35 \mathrm{mrad}$. (C) The density shift measured for each misalignment shown in (A) and (B). ¿From $\Delta \theta$ and $T$, the spread in Rabi frequency $\Delta \Omega$ is calculated. The lines show the expected shift as a function of $\Delta \Omega$ for $T=1 \mu \mathrm{K}$ (solid line) and $3 \mu \mathrm{K}$ (dashed line). The inset shows a zoomed-out plot. (D) For large misalignments, we observe a smaller density shift. This is described using the rotation on the Bloch sphere. As an example, two different values of $\Delta \Omega$ are shown. On each sphere, the average excitation fraction is shown with a solid line, and the spread is indicated by the dotted lines. For small misalignments, we have a small spread in Rabi frequencies. As the misalignment increases, the spread crosses the equatorial plane of the Bloch sphere. At $50 \%$, the sign of the density shift changes, and therefore the portion of the spread centered around this plane averages to zero. The measured density shift is then reduced. 


\title{
Supporting online material for Probing Interactions between Ultracold Fermions
}

\author{
G. K. Campbell ${ }^{1}$, M. M. Boyd ${ }^{1}$, J. W. Thomsen ${ }^{1}$, M. J. Martin ${ }^{1}$, \\ S. Blatt ${ }^{1}$, M. D. Swallows ${ }^{1}$, T. L. Nicholson ${ }^{1}$, T. Fortier ${ }^{2}$, \\ C. W. Oates ${ }^{2}$, S. A. Diddams ${ }^{2}$, N. D. Lemke ${ }^{2}$, P. Naidon ${ }^{3}$, \\ P. Julienne ${ }^{3}$, Jun $\mathrm{Ye}^{1 *} \&$ A. D. Ludlow ${ }^{1}$ \\ ${ }^{1}$ JILA, National Institute of Standards and Technology and University of Colorado \\ Department of Physics, University of Colorado, Boulder, Colorado 80309-0440, USA \\ ${ }^{2}$ Time and Frequency Division, National Institute of Standards and Technology, \\ Boulder, CO 80302, USA \\ ${ }^{3}$ Atomic Physics Division and Joint Quantum Institute, \\ National Institute of Standards and Technology, \\ 100 Bureau Drive Stop 8423, Gaithersburg, Maryland 20899-8423 \\ *To whom correspondence should be addressed; E-mail: junye@jilau1.colorado.edu.
}

\section{Materials and methods}

To first cool and trap the atoms, ${ }^{87} \mathrm{Sr}$ is loaded into a magneto-optical trap (MOT) from a slowed atomic beam. Fig. S1a shows a diagram of relevant energy levels used in cooling, trapping and detecting ${ }^{87} \mathrm{Sr}$. The MOT is operated on the strong ${ }^{1} \mathrm{~S}_{0}-{ }^{1} \mathrm{P}_{1}$ transition, and cools the atoms to millikelvin temperatures. A second MOT stage operated on the narrow ${ }^{1} \mathrm{~S}_{0}-{ }^{3} \mathrm{P}_{1}$ transition is then used to cool the atoms further to microkelvin temperatures. This second stage MOT uses dual-frequency narrow line cooling $(1,2)$. During the MOT phase the one-dimensional optical lattice is superimposed on the atoms in the vertical direction. After the second stage MOT, the MOT cooling beams and magnetic gradient are turned off, leaving 2000 atoms trapped in the 
optical lattice. After the lasers and magnetic fields are turned off, the temperature of atoms remaining in the lattice is $\sim 3 \mu \mathrm{K}$.

To vary the temperature of the atoms, additional cooling or heating is performed on the lattice-trapped atoms. The cooling is performed in three dimensions, with Doppler cooling applied along the transverse direction of the lattice, and sideband cooling (heating) along the longitudinal axis. The laser beams used for cooling (heating) are red (blue) detuned from the ${ }^{1} S_{0}(\mathrm{~F}=9 / 2)-{ }^{3} P_{1}(\mathrm{~F}=11 / 2)$ transition. $\vec{F}=\vec{I}+\vec{J}$ is the total angular momentum, with $\vec{I}$ the nuclear spin and $\vec{J}$ the total electron angular momentum. The cooling (heating) beams are applied first along the transverse direction for $20 \mathrm{~ms}$, followed by $30 \mathrm{~ms}$ along the longitudinal direction. Simultaneous with the sideband cooling (heating) the atoms are spin-polarized in a weak magnetic bias field $(\sim 30 \mathrm{mG})$ using an optical pumping beam resonant with the ${ }^{1} \mathrm{~S}_{0}(\mathrm{~F}$ $=9 / 2)-{ }^{3} P_{1}(F=9 / 2)$ transition. By choosing the correct polarization for the optical pumping beam, atoms are polarized into either the $m_{F}=+9 / 2\left(m_{F}=\right.$ magnetic quantum number) or $m_{F}$ $=-9 / 2$ ground state. To measure the final temperature of the atoms, two different techniques are used. From time-of-flight analysis the temperature of both the longitudinal and transverse directions can be directly measured. By examining the spectral components of sideband spectroscopy as shown in Fig. 1A, the longitudinal temperature can also be accurately extracted. The total atom number is determined by fluorescence measurements. The average density is then calculated using the trap volume. The trap volume is determined using our measured trap frequencies and temperatures and assumes a Maxwell-Boltzmann distribution.

Orienting the lattice in the vertical direction breaks the energy degeneracy between lattice sites, strongly prohibiting inter-site tunneling (3). This ensures that collisions occur only between atoms in the same lattice site. The lattice is aligned with a slight angle with respect to gravity, to accommodate the vertical MOT beams. The probe beam used for spectroscopy is aligned collinear with the optical lattice, and the polarization of the beam is also parallel to that 
of the lattice. To minimize inhomogeneity due to probe beam focusing, the waist of the probe beam is $\sim 5$ times larger than the waist of the lattice focus.

\section{Supporting text}

For a given atom in the electronic ground state ${ }^{1} \mathrm{~S}_{0}$ and motional state $\left(n_{x}, n_{y}, n_{z}\right)$, the excitation probability during the spectroscopy pulse is

$$
p_{e}(\Delta, t)=\frac{\Omega_{\vec{n}}^{2}}{\Omega_{\vec{n}}^{2}+\Delta^{2}} \sin ^{2}\left(\frac{t \sqrt{\Omega_{\vec{n}}^{2}+\Delta^{2}}}{2}\right),
$$

where $\Delta$ is the detuning of the clock laser from resonance, $t$ is the length of the spectroscopy pulse, and the modified Rabi frequency is given by (4):

$$
\Omega_{\vec{n}}=\Omega_{n_{x}, n_{y}, n_{z}}=\Omega_{0} e^{\left(-\eta_{x}^{2}-\eta_{y}^{2}-\eta_{z}^{2}\right) / 2} L_{n_{x}}\left(\eta_{x}^{2}\right) L_{n_{y}}\left(\eta_{y}^{2}\right) L_{n_{y}}\left(\eta_{y}^{2}\right)
$$

Here $\Omega_{0}$ is the bare Rabi frequency, $L_{n_{x, y, z}}$ are Laguerre polynomials, and $\eta_{x, y, z}$ are the LambDicke parameters for the transverse and longitudinal directions (assuming the projection of the misalignment on the transverse plane bisects $\mathrm{x}$ and $\mathrm{y}$ axes):

$$
\begin{aligned}
& \eta_{x}=\eta_{y}=\frac{\sin (\Delta \theta)}{\sqrt{2} \lambda_{L}} \sqrt{\frac{h}{2 m \nu_{x}}} \\
& \eta_{z}=\frac{1}{\lambda_{L}} \sqrt{\frac{h}{2 m \nu_{z}}} .
\end{aligned}
$$

To calculate the density shift, we return to our two-atom model. As discussed in the main text we can explicitly calculate $G_{12}^{(2)}$ from the anti-symmetrized overlap of the two wavefunctions. The antisymmetrized wavefunction $\left|\Psi_{A S}\right\rangle$ is given by

$$
\begin{aligned}
\left|\Psi_{A S}\right\rangle & =\frac{1}{\sqrt{2}}\left(\psi_{1} \psi_{2}-\psi_{2} \psi_{1}\right) \\
& =\frac{1}{\sqrt{2}}((\alpha|g\rangle+\beta|e\rangle)(\gamma|g\rangle+\delta|e\rangle)-(\gamma|g\rangle+\delta|e\rangle)(\alpha|g\rangle+\beta|e\rangle))
\end{aligned}
$$


and

$$
\begin{aligned}
G_{12}^{(2)}(\alpha(t), \beta(t), \gamma(t), \delta(t)) & =\left\langle\Psi_{A S} \mid \Psi_{A S}\right\rangle \\
& =1-\left|\alpha \gamma^{*}+\beta \delta^{*}\right|^{2}
\end{aligned}
$$

Each atom has a slightly different $\Omega_{\vec{n}}$, as given in Eq. S2, and

$$
\begin{aligned}
\alpha(t) & =i \frac{\Omega_{1}}{\Omega_{R 1}} \sin \frac{\Omega_{R 1} t}{2} \\
\beta(t) & =\cos \frac{\Omega_{R 1} t}{2}+i \frac{\Delta}{\Omega_{R 1}} \sin \frac{\Omega_{R 1} t}{2} \\
\gamma(t) & =i \frac{\Omega_{2}}{\Omega_{R 2}} \sin \frac{\Omega_{R 2} t}{2} \\
\delta(t) & =\cos \frac{\Omega_{R 2} t}{2}+i \frac{\Delta}{\Omega_{R 2}} \sin \frac{\Omega_{R 2} t}{2},
\end{aligned}
$$

where $\Omega_{R 1,2}=\sqrt{\Omega_{1,2}^{2}+\Delta^{2}}$ is the generalized Rabi frequency. The inhomogeneity is a consequence of the spread in Rabi frequencies $\Omega_{n_{x}, n_{y}, n_{z}}$, due to the excitation of different motional states. We can characterize this by defining an average Rabi frequency $\bar{\Omega}$ and its RMS spread $\Delta \Omega$,

$$
\begin{aligned}
\bar{\Omega} & =\sum_{n_{x}} \sum_{n_{y}} \sum_{n_{z}} q_{x}\left(n_{x}\right) q_{y}\left(n_{y}\right) q_{z}\left(n_{z}\right) \Omega_{n_{x}, n_{y}, n_{z}} \\
\Delta \Omega^{2} & ={\overline{\Omega^{2}}}^{2}-\bar{\Omega}^{2} .
\end{aligned}
$$

Here $q_{x, y, z}\left(n_{x, y, z}\right)$ is the normalized Boltzmann factor. The inhomogeneity in the Rabi frequencies $(\Delta \Omega)$ is affected by $T$ through the Boltzmann factor and by $\Delta \theta$ through the probe misalignment. To approximate the average density shift for the entire atomic ensemble, we set $\Omega_{1}=\bar{\Omega}+\Delta \Omega$ and $\Omega_{2}=\bar{\Omega}-\Delta \Omega$ in Eq. S8 for our two-atom model. At a time $t$ during the spectroscopy pulse, the atoms experience an ensemble-averaged shift:

$$
\Delta \nu(t)=\frac{2 \hbar a_{g e}}{m} G_{12}^{(2)}(\bar{\Omega}+\Delta \Omega, \bar{\Omega}-\Delta \Omega)\left(\rho_{g}-\rho_{e}\right) .
$$


This shift evolves during the spectroscopy pulse, and for the final density shift we time-average $G_{12}^{(2)}$ over the total pulse length $t_{F}$ as

$$
\Delta \nu_{\text {ave }}=\frac{1}{t_{F}} \int_{0}^{t_{F}} \Delta \nu(t) d t .
$$

\section{References}

S1. T. H. Loftus, T. Ido, A. D. Ludlow, M. M. Boyd, J. Ye, Phys. Rev. Lett. 93, 073003 (2004).

S2. G. K. Campbell, et al., Metrologia 45, 539 (2008).

S3. P. Lemonde, P. Wolf, Physical Review A 72, 033409 (2005).

S4. D. J. Wineland, W. M. Itano, Phys. Rev. A 20, 1521 (1979). 
A

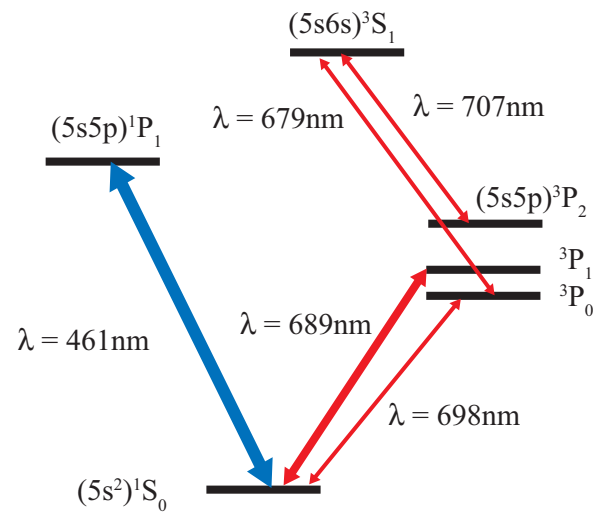

B

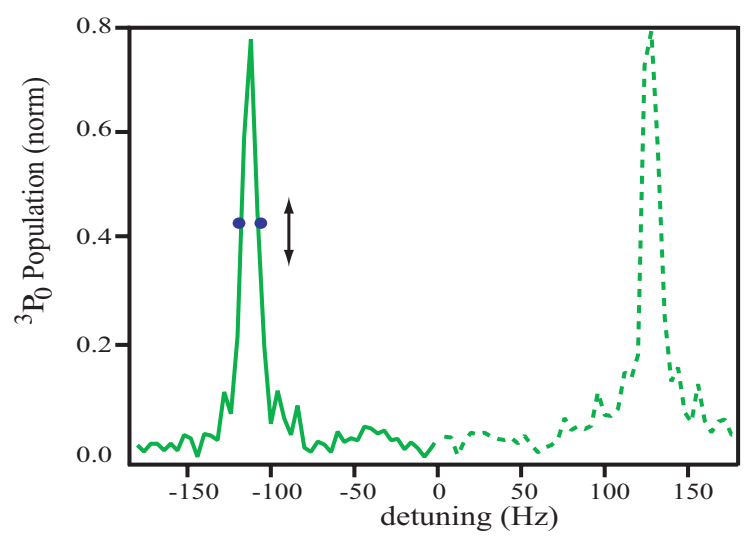

Figure S1: Experimental setup for the ${ }^{87} \mathrm{Sr}$ optical clock. (a) Relevant energy levels for laser cooling and trapping are shown. The first MOT stage is operated on the strong transition at 461 nm. During this MOT stage atoms that are lost to the ${ }^{3} \mathrm{P}_{2}$ and ${ }^{3} \mathrm{P}_{0}$ states are pumped back to the ${ }^{1} \mathrm{~S}_{0}$ state using two repump beams at $679 \mathrm{~nm}$ and $707 \mathrm{~nm}$. The second MOT is operated on the narrow $689 \mathrm{~nm}$ transition, and the wavelength of the clock laser used for spectroscopy is $698 \mathrm{~nm}$. After the experiment is performed, atoms in the ${ }^{1} \mathrm{~S}_{0}$ state are detected by measuring fluorescence on the strong ${ }^{1} \mathrm{~S}_{0}-{ }^{1} \mathrm{P}_{1}$ transition. Atoms that have been excited to ${ }^{3} \mathrm{P}_{0}$ by the clock laser pulse are transferred back to the ${ }^{1} \mathrm{~S}_{0}$ using the two repumping beams, and are again measured by fluorescence. (b) Two sample spectra. Here, ${ }^{87} \mathrm{Sr}$ atoms are successively polarized into either the $m_{F}=9 / 2$ or $-9 / 2$ state. The separation between the two spectroscopy lines is $\sim 250 \mathrm{~Hz}$. During the actual experiment, we polarize the atoms to each pure state successively, and lock near the full-width at half-maximum of the peak (indicated by dots). By moving these lock points the desired excitation fraction is selected. 